\title{
POSITRONIUM CHEMISTRY
}

Final Report

S. J. Tao

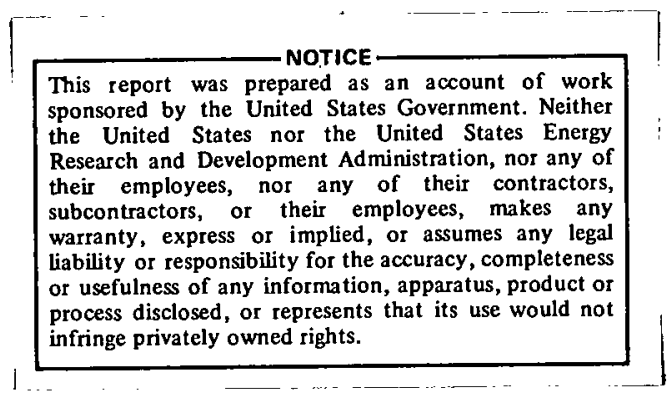

The New England Institute

Ridgefield, Connecticut 06877

February, 1966 - January, 1975

PREPARED FOR THE U.S. ATOMIC ENERGY COMMISSION

UNDER CONTRACT NO. AT (11-1) -3373 


\section{DISCLAIMER}

This report was prepared as an account of work sponsored by an agency of the United States Government. Neither the United States Government nor any agency Thereof, nor any of their employees, makes any warranty, express or implied, or assumes any legal liability or responsibility for the accuracy, completeness, or usefulness of any information, apparatus, product, or process disclosed, or represents that its use would not infringe privately owned rights. Reference herein to any specific commercial product, process, or service by trade name, trademark, manufacturer, or otherwise does not necessarily constitute or imply its endorsement, recommendation, or favoring by the United States Government or any agency thereof. The views and opinions of authors expressed herein do not necessarily state or reflect those of the United States Government or any agency thereof. 


\section{DISCLAIMER}

Portions of this document may be illegible in electronic image products. Images are produced from the best available original document. 


\section{Table of Contents}

I. Introduction

II. Experimental Methods

1. Positron Annihilation Lifetime Measurement

2. Two Gamma-Ray Angular Correlation Measurement

3. Doppler Broadening or Line-Shape of the Two Gamma-Ray Annihilation

4. Three Gamma-Ray Coincidence

5. Mono-Energetic Slow Positron Beam Studies

6. Other Methods

III. Brief Summary of Present Status

1. Positron-Atom or -Molecule Collision Problems

2. Positron and Positronium Compounds

3. The Formation of Positronium

4. The Properties of Positronium

5. Positrons in Metallic Media

6. Positrons in Ionic Solids

7. Positrons in Polymers

8. Positrons and Positron Annihilation and Phase Transitions

IV. Work Performed

1. Gaseous Media

2. Polymers and Macromolecules

3. Molecular Liquids and Their Solutions

4. Phase Transitions

5. Metals

6. Positronium Chemical Reactions

7. Formation of Positronium

8. Positrons and Positronium in Porous Solids

9. Data Reduction

10. Instrumentation

11. Data Compilation 
V. Future Outlook and Recommendations

VI. References

VII. List of Publications 


\section{Introduction}

Positron annihilation, or the interaction between slow positrons and matter, has been an intriguing and interesting topic in the physical sciences since the existence of positrons was theoretically predicted by Dirac $^{1}$ and later discovered in cosmic showers. ${ }^{2}$

Since the positron is the anti-particle of the electron, the common species of the electron-positron pair in our world, positrons are only stable in a place where electrons are absent. A positron will annihilate with an electron and change its mass into energy in the form of gamma-rays. Positron behavior has been found to obey the rules of ordinary physics, including quantum mechanics and quantum electrodynamics. The annihilation process depends on the configuration of electrons in the matter. As a matter of fact, the predictable behavior of positrons and positron-electron annihilation was one of the great triumphs of modern theoretical physics. Therefore, naturally, positron annihilation has been used as a means of studying the properties of materials as well as a test of the fundamental theories of physics.

A positron can also bind with an electron to form a hydrogen-like atom, called positronium. ${ }^{3}$ This is one of the important processes of positron-electron interaction and annihilation in molecular substances. The positronium (PS) formed will be in either the para-state with $S=0$, or the ortho-state with $S=1$. In a free space the $p-P s$ will annihilate with a mean life of 0.125 nsec by emitting two gamma-rays in opposite directions, and the o-Ps will annihilate with a mean life of 140 nsec by emitting three gamma-rays in a plane. Because of the long mean life of o-Ps, even in con- 
Table 1 A Brief Outline of the Methods Used

for the Studies on Positron Annihilation

Experimental

Methods

Annihilation

lifetime

Angular

Correlation

Doppler Broadening

$3-\gamma$ coincidence

Mono-energetic

slow $e^{+}$beam
Apparatus

Form of Data

Affecting Factors

Applications

Fast scintillation counters and

time-to-amplitude converter

Scintillation

counters and moderate

fast electronics

Ge (Li) de tector and stable electronics

Similar to angular correlation

Similar to annihilation lifetime in addition

to beam apparatus

\section{Annihilation events} vs. time in nanosecond and subnanosecond range

Annihilation events

$(2-\gamma)$ vs. angular derivation, resolution $<1 \mathrm{mrad}$.

Line shape of the $(2-\gamma)$ $0.51 \mathrm{MeV} \gamma$-rays. resolution, $\sim 2 \mathrm{keV}$

Coincidence rate of $3-\gamma$ events

\section{change in beam} intensity at a certain energy
Density of

formation of Ps.

Density of electrons with opposite spin in momentum space, anisotropic

Density of electrons with opposite spin in energy space

Density of electrons and formation of Ps.

Scattering and annihilation of $e^{+}$ electrons and
Reactions of $\mathrm{e}^{+}$and $\mathrm{Ps}$

Momentum distribution of $\mathrm{e}^{+}$, reactions of $\mathrm{p}-\mathrm{Ps}$.

As above, but still limited by its resolution

Reaction of $\mathrm{e}^{+}$ and Ps

Scattering and annihilation cross sections 
densed media, o-Ps may possess a mean life long enough to undergo fast chemical reactions with its surrounding substances.

In any medium a large fraction of positrons will annihilate with electrons when a positron passes through the electron cloud without forming a "bound" state. Such a process is called "free" or "direct" aninihilation.

Many review articles have been written about positron annihilation or positronium chemistry. Some of them are cited here. ${ }^{3-17}$

\section{Experimental Methods}

The several experimental methods which are commonly used to study positron annihilation will be described here. Some of the features of these methods are summarized in Table 1.

\section{Positron Annihilation Lifetime Measurement}

This method takes advantage of a special decay scheme of some positron emitting radioactive isotopes, such as the commonly used Na22. After a positron is emitted, Na22 decays into a short lived $\left(t_{1 / 2} \simeq 10^{-12}\right.$ sec $)$ excited state of $\mathrm{Ne} 22$ which, in turn, decays to its ground state by emitting a $1.28 \mathrm{MeV}$ gamma-ray. Therefore, the $1.28 \mathrm{MeV}$ gamma-ray can be used to mark the birth of a positron and the gamma-rays produced by annihilation to mark the death of a positron. These gamma-rays can be detected by a pair of fast gamma-counters, generally scintillation counters, one for the $1.28 \mathrm{MeV}$ gamma-rays and another for the annihilation gamma-rays. The time durations between the arrival times of these two kinds of gamma-rays detected are converted electronically into the heights of electrical pulses. ${ }^{18,19}$ The pulses are fed into a multi-channel analyzer and stored. The resultant picture is in the form of annihilation events against time, and is called 
a positron annihilation lifetime spectrum. In the lifetime spectrum, annihilation of o-Ps will generally appear as a long lifetime component; free positrons, an intermediate lifetime component; and P-Ps, à very short lifetime component. The measured annihilation rate (inverse of the mean life) is a function of the electron density with an opposite spin to the positron at the position of the positron.

2. Two-Gamma-Ray Angular Correlation Measurement

This method measures the angular deviation with respect to the exact opposite direction of the two gamma-rays emitted by the $S=0$ mode of annihilation. The angular deviation comes from the non-zero kinetic energy possessed by the annihilating positron-electron pair. For free positron annihilation, the positrons are nearly thermalized. Therefore, nearly all the energy comes from the energy possessed by the electrons. This will give the momentum distribution of the electrons annihilating with positrons in the medium. For p-Ps annihilation, a very narrow peak appears. Obviously, annihilation by the $S=1$ mode can hardly be detected. Slots of several shapes can be employed for the two scintillation counters. The long slot configuration is the one commonly used because of better counting efficiency. 20,21 The momentum distribution of the "free electrons" in a metal will appear as a bell-shaped curve if a long slot configuration is used. Sometimes, a point slot or cross slot configuration has also been used. $^{22}$ Very fast subnanosecond electronics is not necessarily required for this method. The measured curve is a function of the electron density in the momentum space at the position of the positron. Anisotropic effects can be detected by this method. 
3. Doppler Broadening or Line-Shape of the Two Gamma-Ray Annihilation A Ge(Li) solid state detector with high energy resolution is used in this method. ${ }^{23}$ The broadening, or energy deviation, of the gamma-rays to the exact energy produced by the annihilation of the positron-electron pair via the $S=0$ mode at rest is measured. The actual energy deviation or the line-shape of the annihilation gamma rays can be obtained by deconvoluting the energy resolution function of the detector out from the measured curve. The advantage of this method is the speed of the measurement. At present, the resolution of the Ge(Li) detector is still not as high as the one being obtained by the method of angular correlation.

\section{Three Gamma-Ray Coincidence}

Three gamma-ray detectors placed in the same plane as the positron: source and sample are used. Usually, the three detectors are placed at $120^{\circ}$ to each other. The coincidence rate of the annihilation via the $\mathrm{S}=1$

mode is measured. ${ }^{24}$ Since only the annihilation of o-Ps produces a signif-. icant amount of coincident three gamma-rays, this method is not very sensitive in detecting the annihilation of free positrons and not effective in detecting the annihilation of p-Ps.

\section{Mono-Energetic Slow Positron Beam Studies}

The successive production of a mono-energetic slow positron beam... has been achieved only recently. ${ }^{25,26}$ After high energy positrons hit into a solid, a small fraction of them may emerge from the surface of the solid with a nearly mono-energetic distribution at an energy of several electron volts. The energy distribution and yield depend on the properties of the solid and the preparation of the surface. A nearly mono-energetic slow 
positron beam can be obtained by using the above phenomenon with a suitable experimental set-up. The energy and intensity of the beam can be measured by the method of time-of-flight. The timing instrument is similar to the one used by the method of annihilation lifetime measurement.

On the other hand, the energy spectrum of the slow positrons emitted from the solid surface can be used to study the properties of the solid.

6. Other Methods

The peak-to-valley ratio of the annihilation gamma-ray spectrum measured by a $\mathrm{NaI}(\mathrm{TI})$ scintillation counter was also used as a means of measuring the relative change of the ratio of the two gamma-ray to three gamma-ray annihilation in the early days. 27

Before the availability of multi-channel analyzers, the method of delayed coincidence counting by varying the length of delay times was used for lifetime measurements. 28

The method of angular correlation can be used in conjunction with the method of lifetime measurement. ${ }^{29}$ The measurement of positron annihilation lifetime spectra at various energies of the annihilation gamma-rays is another possibility. Other kinds of set-ups are also possible.

\section{Brief Summary of Present Status}

1. Positron-Atom or -Molecule Collision Problems

The discovery of a shoulder in the positron annihilation lifetime spectra in argon and other noble gases suggested a changing annihilation rate and a minimum in scattering cross sections for slow positrons 30,31 similar to the Ramsauer minimum observed for slow electrons. The experimental information obtained by the method of positron annihilation lifetime mea- 
surement cannot provide an accurate and precise profile for both the annihilation and scattering cross sections at the same time. This has hindered the progress of the theoretical work in the calculation of collision cross sections of various simple gases for slow positrons because of the lack of experimental data to check the validity of the theories or approximations used.

However, the progress in the mono-energetic slow positron beam studies has changed the picture recently. ${ }^{32,33}$ At present, there is still a slight difficulty in obtaining a mono-energetic positron beam with energies lower than $2 \mathrm{eV} .{ }^{34}$ Once this difficulty is overcome the determination of the experimental total scattering cross sections at such lower energies can be : used to check the values calculated theoretically. 35

If the (total) scattering cross sections of a gas for positrons are known, it will be relatively easy to calculate the annihilation rate. or cross sections at various energies using the experimental data obtained from the lifetime measurement. From the results obtained by measuring positron annihilation lifetime spectra in gases under an electric field, one may be able to evaluate the positronium formation cross section and the slowing down process of positron annihilation. This will help to interpret precisely the appearance of a peak at the end of the shoulder for free positron annihilation in some dense noble gases at low temperatures. 36

The work in this field will increase our fundamental knowledge in the collision problems and reaction mechanisms in the energy range of chemical energies.

\section{Positron and Positronium Compounds}

The high values of $Z_{\text {eff }}$ of some gases, such as Freon-12 $\left(\mathrm{CCl}_{2} \mathrm{~F}_{2}\right)^{37}$ 
and $\mathrm{NH}_{3}{ }^{38}$, for free positron annihilation have been attributed to the formation of some positron compounds or complexes. Similarly, the high annihilation rates for positronium in certain chemically reactive gases, such as $\mathrm{Br}_{2}, \mathrm{Cl}_{2}$ and $\mathrm{NO}_{2}$, have also been attributed to the formation of some positronium compounds, such as PsBr. 39,40 .

In liquid media, the formation of positronium compounds or positronium charge-exchange complex compounds has been suggested as the cause of high quenching rates in solutions of iodine, p-benzoquinone and many nitrohydrocarbons. 41,42

On the theoretical side, the work on the calculation of the binding energies of either positron or positronium compounds has proceeded rather slowly. The slow progress stems from the difficulty in performing this kind of calculation. Because of the light mass of positrons, the BornOppenheimer approximation is not valid, or we cannot treat the positron as a heavy center of mass moving slowly with the electron cloud moving swiftly around it. We have to treat the positron as a fast moving particle like an electron. One of the models is to treat the positron as an electron moving around in atomic orbitals. It is hoped more research will be performed in this area.

\section{The Formation of Positronium}

A positronium atom can be formed when a slow positron captures an electron during a collision with an atom or a molecule. By conservation of energy, the energy of the positron must be higher than the threshold energy, $E_{t h r}=I_{A}-I_{p}$, where $I_{p}$ is the ionization potential of the positronium $6.8 \mathrm{eV}$, and $\mathrm{I}_{\mathrm{A}}$ is the ionization potential of the colliding atom or molecule. In a gaseous medium, any positronium atom formed with a 
kinetic energy higher than $I_{p}$ will not be stable and will be easily dissociated upon subsequent collisions. Therefore, there is an upper energy limit of $I_{A}$ for positrons to be able to form positronium. For some noble gases the lowest excitation energy, $E_{\text {exc }}$, lies above $E_{t h r}$. Therefore, there is an energy region, $E_{\text {exc }}>E>E_{t h r}$, where slow energy decrement elastic collisions are the only slowing down mechanism for positrons. This, in turn, favors the formation of positronium in this energy region. The above conditions constitute the principles of the we11-known "Ore gap" theory. 43

In a condensed medium, the situation is different. The exact values of the ionization potential cannot be measured. The slow positrons and the positronium formed are not entirely free. In addition, for most of the experiments the positrons are ejected into the medium at energies much higher than several tens of electron volts: Or, the positrons are a source of ionization radiation themselves. Therefore, there is a possibility that, when a positron is nearly thermalized, a nearly thermalized electron may also be nearby. If the electron is under the coulombic influence of the positron, a positronium atom may form. This constitutes the foundation of the "Spur" theory for positronium formation. 44

The values of scattering cross sections of some gases for positrons measured by the mono-energetic positron beam studies certainly will help us to evaluate more quantitatively the mechanism of the formation of positronium in gaseous media.

The "Spur" theory is an interesting model for the explanation of the formation of positronium or a part of positronium in condensed substances. More studies, theoretically to make the "Spur" theory a more 
quantitative one, and experimentally to provide more data for the quantitative interpretation of the theory, will not only solve the problem of how positronium is formed but also many problems of reaction mechanisms and processes in radiation chemistry.

4. The Properties of Positronium

In studying the properties of positronium, we are usually concerned with o-Ps because it possesses a longer mean life and can be readily detected. Many of the properties of p-Ps will be similar to those of o-Ps, except we have to consider its different spin and shorter mean life.

In a medium where no substance with unpaired electrons in their structures are present, o-Ps will not change its spin state after a col1ision. However, during any collision there is a chance for the positron in o-Ps to sense an electron with an opposite spin and annihilate with it. This process will reduce the mean life of o-Ps and is called "pick-off. quenching." In a medium where substances with unpaired electrons in their structures are present, o-Ps may change to $\mathrm{p}-\mathrm{Ps}$ (and $\mathrm{p}-\mathrm{Ps}$ may change to o-Ps) after a collision. This process will reduce the mean life of o-Ps and is called "conversion quenching." In a medium where chemically reactive substances are present, o-Ps may react by either forming a positronium compound, a complex, or being oxidized. This process will also reduce the mean life of o-Ps and is called "chemical quenching."

The plck-off quenching rates of many liquid organic compounds were found to relate linear $1 \mathrm{y}$ to their electron polarizabilities, for each series of homologues. ${ }^{45}$ Later a more thorough study by us found that pickoff quenching rates in liquid organic compounds are related to inter- 
and intra-molecular forces of the molecules around the o-Ps atom. ${ }^{46}$ This is manifested by the simple relationship between the pick-off quenching rate and the surface tension of the liquid medium. In terms of cross sections, assuming full thermalization of positronium, the values of the pickoff quenching cross sections have been found to be around the order of $10^{-21} \mathrm{~cm}^{2}$.

A typical conversion quenching agent is oxygen gas. ${ }^{40}$. Many paramagnetic salts are classified as conversion quenching agents in aqueous solutions. However, the presence of unpaired electrons in the molecular structure is not a sufficient condition for identifying a conversion quenching agent. Because the chemical activity of a compound towards positronium may be very strong, a chemical reaction will take place before conversion reaction can proceed. A typical case here is nitrogen dioxide gas. 39 Nitrogen dioxide was known to be a very strong quenching agent and was suspected to be a conversion quenching agent. Recent experimental work by us has proved beyond a doubt that nitrogen dioxide quenches positronium by chemical reaction. The values of cross sections of conversion quenching agents have been found to be around the order of $10^{-19} \mathrm{~cm}^{2}$ and the values of cross sections of chemical quenching agents have been found to be higher than $10^{-19} \mathrm{~cm}^{2}$ and can be more than $10^{-16} \mathrm{~cm}^{2}$.

In a solution consisting of an inert (pick-off quenching only) solvent and an active quenching agent as a solute, the reaction rate is found to be diffusion-controlled, or strongly influenced by the viscosity of the solution. ${ }^{41}$ The values of the activation energies for various quenching agents have been found to be around the values of the activation energy of the viscosity of the solvent. However, deviations from the Arrhenius law at higher energies have been found. $41,42,47$ This has been 
interpreted as due to the intrinsic reaction mechanism, non-thermalization of o-Ps, or the formation of charge transfer complexes.

More work in this area certainly is needed. This will broaden our present knowledge of free radical reactions as well as positronium reactions.

\section{Positrons in Metallic Media}

Metallic substances have been the major focus of past and present research activities in positron annihilation studies. ${ }^{15,16}$ Using the method of angular correlation, the electron density in the momentum space can be readily obtained for the "free" or conduction electrons. This method has proved to be very sensitive to many factors, such as the orientation, order and disorder, purity, defects, temperature, history of treatment, etc. Recently, the method of lifetime measurements and Doppler broadening have also been commonly used, not so much in the interest of pure fundamental research as in the direction of using positron annihilation as a testing tool in applied sciences. Seeger has pointed out that positron annihilation is a very sensitive method for the detection of the concentration of thermal vacancies in some metals in the concentration range of $10^{-7}$ to $10^{-4} \cdot 16$

At present, in pure fundamental research the major direction is in: (1) the development of new theories, such as the pseudo-potential theory and the renormalized free atom model, in addition to the band structure theory; and (2) the calculation of the Compton profile. On the more applied side, work is centered on: (1) the effects of various defects: and (2) transitions, such as ferromagnetic transition, glass transition, etc., in metals.

6. Positrons in Ionic Solids

The interests and direction of the research are much the same as 
(5) Positrons in Metallic Solids.

7. Positrons in Polymers

The sensitivity of positron annihilation, particularly the annihilation lifetime spectrum, to various changes, composition, structure, crystallinity, radiation damage, etc., in organic polymers has been amply demonstrated in the past. $17,48,49$ Recently, similar results have been found for inorganic glasses 50,51 and organic proteins. ${ }^{52}$ For most of these property changes, the method of positron annihilation is comparable to other methods commonly used. However, for temperature changes, such as glass transition, the method of positron annihilation has been found to be more sensitive than other methods. 53,54 More work in this field certainly'will increase our present knowledge of the mechanisms involved in glass transition and other transitions in polymers.

8. Positron and Positronium Annihilation and Phase Transitions The change of positron annihilation processes, particularly the ones detected by the annihilation lifetime measurement, around the phase transitions, glass transition, second order transitions and other transitions are well known. In general, both the mean life and the intensity of the lifetime component change abruptly during the transition. The liquidsolid and solid (isotropic)-solid (anisotropic) transitions of methanol, deuteriated methanol and methyl mercaptan measured by us 55,56 and the transitions of liquid crystals measured by Walker and his colleagues 57,58 are excellent examples. However, there is a lack of theoretical work to interpret these changes. For example, we are still unable to answer why the glass transition point of nylon 06 measured by positron annihilation 
lifetime spectra is roughly $15^{\circ} \mathrm{C}$ lower than those measured by conventional methods. More work in this area will certainly help our present understanding of the theory of molecular solids.

IV. Work Performed

(If $L$ precedes the index number, please find the cited paper in VI List.)

1. Gaseous Media

Our work in this area has been directed toward gathering the following information: (i) positronium reaction to chemically active gases, (ii) annihilation of positrons during slowing down, and (iii) the values of scattering and annihilation cross sections.

We have studied $\mathrm{Br}_{2}, \mathrm{O}_{2}$, NO and $\mathrm{NO}_{2}\left(\mathrm{~N}_{2} \mathrm{O}_{4}\right)$ and found $\mathrm{Br}$ and $\mathrm{NO}_{2}$ to be strong quenching agents for o-Ps and $\mathrm{O}_{2}$ and NO to be only a moderate quenching agent. L38, L45, L53, L55 we have also found that the strong quenching behavior of No found by earlier workers is surely due to the contamination of $\mathrm{NO}_{2} \cdot{ }^{\mathrm{L} 45}$ Additional work using the method of angular correlation has confirmed $\mathrm{NO}_{2}$ to be a chemical quenching agent instead of a conversion quenching agent, as generally believed for a long time, and $\mathrm{O}_{2}$ to be principally a conversion quenching agent. L53,L55

Many positron annihilation lifetime spectra in pure helium, argon and nitrogen at various densities up to 50 amagats have been measured. L14, L19, L23, L26 Free positron annihilation rates for these gases have been found to be nearly linearly dependent on the density of the gas.

A small shoulder was discovered by us in the annihilation lifetime spectra for nitrogen at lower densities. ${ }^{26}$ This indicates that even in nitrogen free positrons annihilate during the slowing down process. A 
study based upon the kinetic theory was made to evaluate the values of the scattering cross section and the annihilation cross section of free positrons in helium. ${ }^{\text {L14 }}$ It has been pointed out that a minimum in the scattering cross sections, just above the thermal energy, is a necessary condition for giving a well defined shoulder at room temperature and a second peak on the shoulder at lower temperatures for helium.

We have also tried to calculate the fraction of Ps formed in helium using the available theoretical values of cross sections. ${ }^{444}$

2. Polymers and Macromolecules

The interests here are centered on the possibility of using positron annihilation as a tool for the study of the various properties of polymers and macromolecules. We studied nitric acid etched polypropylene and were the first to discover that in a porous medium with the interpore distances in the order of $100 \AA$ or less, such as the nitric acid etched polypropylene, there is a chance for o-Ps to move out from the solid phase and into the pores where o-Ps appears as a very long lifetime component in the positron: annihilation lifetime spectrum. ${ }^{215, L 31}$ Using polypropylene of various molecular weights as examples, we proved that the average positron or positronium annihilation rate in a medium with a non-homogeneous micro-structure is a constitutive property of the volume fraction of the various subphases. ${ }^{25, L 47}$ Our work on positron annihilation in nylon 06 during the glass transition has shown that positron annihilation is a very sensitive tool for the study of the glass transition of polymers. At present, we are working on the glass transitions of polyethylene and alloy polymers.

We also discovered that the annihilation rate of the long lifetime component is nearly linearly dependent on the critical surface tension of 
the polymer medium. ${ }^{\text {L56 }}$ We have made measurements of the positron annihilation lifetime spectra in amino-acids and proteins. ${ }^{48}$ The annihilation rates have been found to be related to the number of polar groups in the amino acids. These all point to the fact that the annihilation rate of positrons or positronium is a function of the molecular structure of the medium and it is a constitutive property of the molecular structure of the medium. We have also made angular correlation measurements for several amino acids and the results will be summarized in a paper published at a later date.

\section{Molecular Liquids and Their Solutions}

A large amount of time has been spent on both experimental and theoretical studies in this field. The purpose of the work was twofold: (1) to find a general rule for the pick-off quenching rate of o-Ps in molecular liquids, and (2) to understand the reaction kinetics of positronium reactions when a strong quenching agent is present in an "inert" solvent.

We have found a general rule: a relationship between the pick-off annihilation rate and the surface tension of the liquid molecular medium. ${ }^{43}$ Theoretical support has also been worked out for the above rule. ${ }^{16, \text { L17, } 43}$ The relationship between the annihilation rate of the long lifetime component and the critical surface tension of the polymers further supports the generality of the rule. ${ }^{\text {L56 }}$

As for the second purpose, we have established that at a temperature near room temperature, the positronium reaction rate with a dilute quenching agent in an "inert" solvent is nearly diffusion-controlled, or 
it is a function of the viscosity of the solution. L5, L6, L10,L18,L22, L46 Here, as in the case of the relationship between the plck-off annihilation rate and surface tension, the polar oxygenated short chain hydrocarbons behave in a slightly different manner from the other non-polar or less polar compounds. 446 The activation energies of the reactions are found to be about the same as the values of the activation energy of the viscosity of the solvent used. However, cases of non-linearity in the Arrenhius plot have been foumd. We have also offered the non-thermalization of o-Ps as one of the possible explanations for the appearance of non-linearity. ${ }^{\mathrm{L}}$.

\section{Phase Transitions}

The abrupt change of positron annihilation behavior during-a phase transition has been a well-known phenomenon. However, a quantitative or even a satisfactory semi-quantitative explanation for the change has never been achieved. We have made many experimental measurements of the positron annihilation lifetime spectra for various organic compounds during the liquid-solid phase transition. No general trends have been found. For example, both the mean life and the intensity of the long lifetime component in benzene decrease abruptly when the liquid solidifies. ${ }^{\text {L5 }}$ However, if $2 \%$ of nitrobenzene is added to the benzene, no long lifetime component appears in the 1iquid phase, but a long lifetime component appears in the solidified medium. For another example, the intensity of the long lifetime component for positron annihilation in several long chain hydrocarbons and polyglycols at solid state shows a high plateau at the temperatures just below the solidifying point. ${ }^{\text {L52 }}$ Al1 these results are puzzling and need explanation. We suspect that they are related to the migration and reaction of slow positrons in a solid molecular medium. How- 
ever, more experiments are needed. At present, we are using the method of angular correlation to obtain additional information in this respect.

Positron annihilation is also very sensitive to second order transitions. In the section dealing with polymers we have mentioned glass

transitions. $^{\text {L39 }}$ We have studied the glass transition of a glassy metal, PdCuSi. We have also studied the second order transitions of solid methanol, deuteriated methanol and methyl mercaptan. ${ }^{32, L 36, L 52}$ The transitions detected by positron annihilation are sharp and distinctive. A change in $\mathrm{KC1O}_{4}$ was also detected. ${ }^{\mathrm{L} 33}$

5. Metals

Metals have always been the favorite substance for positron studies. In the past most work was conducted using the method of angular correlation. Although one can extract more information about the electron configuration in metals by using the method of angular correlation, this method is tedious to operate. We have tried to use the method of annihilation lifetime measurement to study a few metal samples in order to explore the possibility of using positron annihilation as a practical tool to study the properties of metals. ${ }^{\text {L } 30, L 57, L 59 ~}$ We have found it interesting and useful in certain respects. At present, we are continuing this kind of work under a contract from the Naval Air Systems Command.

\section{Positronium Chemical Reactions}

The work done by us has proved beyond a doubt that positronium does react chemically with many chemically active compounds, such as $\mathrm{Cl}_{2}, \mathrm{Br}_{2}$, $\mathrm{NO}_{2}$, p-benzoquinone, etc. $\mathrm{L} 3, \mathrm{~L} 6, \mathrm{~L} 8, \mathrm{~L} 10, \mathrm{~L} 22, \mathrm{~L} 38, \mathrm{~L} 45, \mathrm{~L} 53$ Most of the positronium reactions are very fast. Therefore, in a solution; the reaction rate is roughly collision controlled or diffusion controlled, and the values of the 
activation energies are very low, $0.1 \mathrm{eV}$ or less. However, we are still not very sure about the exact positronium compounds formed during these reactions. Since the mean life of the positronium compounds are expected to be about $0.4 \mathrm{nsec}$, the formed compounds may still be in their excited state and the annihilation may take place before the de-excitation. More work certainly is required.

\section{Formation of Positronium}

It is generally believed that, in a not-so-dense gaseous medium, positronium may be formed when the following reaction is energetically probable:

$$
e^{+}+A \rightarrow P s+A^{+}
$$

Since a positron or a positronium atom is a light particle during the above reaction, most of the energy will be carried by either $\mathrm{e}^{+}$or Ps. The favorable energy range for positrons to form positronium is $I_{A}>E>I_{P}-I_{A}$, as suggested by ore. ${ }^{43}$ In a condensed medium certainly the above conditions will be modified. However, because we do not have exact knowledge of the ionization potential of various substances in a condensed state, a quantitative description is impossible. Recently, a "Spur" theory was suggested by Morgensen to account for the formation of positronium in a liquid medium. ${ }^{44}$ This was mentioned previously in Section III, 2.

We have studied the "Spur" theory in great detail. A report will appear elsewhere. We shall give a very brief summary here.

Certainly, the recombination between a pair of nearly thermalized positrons and electrons should be one of the processes for the formation of positronium. However, we cannot borrow all the mechanisms involved in the electron-ion recombination reaction in radiation spurs for the positron- 
electron recombination reaction. The process of ionization happens all the way along the track of the primary ionization particle. Therefore, the recombination of electron-ion pairs takes place all the way in the spurs around the track. The recombination of the positron-electron only happens near the place of the last ionization pair produced by the primary particle, the positron. Around the positron, on the average, there is an extra positive charge. In addition, the positron is much lighter than the positive ions produced by ionization. These all will make the recombination of a positron and an electron less probable than the recombination of electrons and ions.

In a condensed medium the mean life of free positrons is only about $0.4 \mathrm{nsec}$, which is much shorter than the mean life of electrons or solvated electrons. In non-polar liquids where the di-electric constant is 2.0 , the mean recombination distance is $200 \AA$. The thermalization time of positrons and the positron-electron recombination time combined is of the order of magnitude of the mean life of free positrons. Therefore, at least part of the positrons may annihilate before forming Ps.

Quantitatively, the simple "Spur" theory cannot explain the change of the intensity of the long lifetime component which is attributed to the annihilation of o-Ps for positron annihilation in the homologues of organic compounds. For example, the intensity, $I_{2}$, is $19 \%$ for methanol, which has a di-electric constant of 33 and the values of $I_{2}$ are all about $20 \%$ for higher homologues of n-alkanols whose di-electric constants decrease with the increase of the length of the carbon chain. From the "Spur" theory we would expect the values of $I_{2}$ to increase with the increase of the length of the carbon chain. 
Therefore, the introduction of the "Spur" theory has not helped our present understanding of the formation of positronium in molecular liquid media much. We think more work, both theoretically and experimentally, is required. The work in this area will add much to our present knowledge of the mechanisms of radiation chemistry because the formation of positronium is a result of irradiation.

8. Positrons and Positronium in Porous Solids

We have studied positron and positronium annihilation in silica gels and silica gels adsorbed with other substances. We have found that it is very useful to use silica gels as a medium for the study of positronium reactions because the mean life of o-Ps in the pores of the silica gels is on the order of several tens of nanoseconds. In addition, a well defined p-Ps narrow peak appears in the angular correlation curves. Using pure silica gels and varying the temperature we were able to study the migration of o-Ps in the solid phase and the behavior of o-Ps in the pores. ${ }^{\text {24, }}$ L28, L37, L40,L51 By adsorbing some materials on the surface of the pores we were able to study the positronium reactions as well as the surface chemistry of the silica ge1s. L21,L37,L53,L55

\section{Data Reduction}

We have studied thoroughly the problems involved in the data reduction for the method of positron annihilation lifetime measurement. We have pointed out the effect of non-linear deviation of the apparatus, as well as the difficulties arising in choosing a criterion of statistical significance. ${ }^{27, L 11, L 34}$ Our programs are used by many workers around the world. Recently, we have also studied three more methods of data reduction used for the de-convolution of the resolution function. The results will 
appear elsewhere and a brief summary is given below.

First is the method of matrix inversion. If the resultant spectrum is $y(t)$, the actual original spectrum is $Y(t)$ and the resolution function is $R\left(t, t^{\prime}\right)$, we have

$$
y(t)=R\left(t, t^{\prime}\right) Y\left(t^{\prime}\right)
$$

where $y$ and $Y$ are column vectors and $R$ is a matrix. If properly constructed, $R$ can be made to be a square matrix, then we have

$$
Y\left(t^{\prime}\right)=R^{-1}\left(t, t^{\prime}\right) y(t) \text {. }
$$

Since $R\left(t, t^{\prime}\right)$ is known, theoretically we can solve $R^{-1}\left(t, t^{\prime}\right)$ and obtain $Y\left(t^{\prime}\right)$. However, practically we have found that the errors involved in solving $R^{-1}$ are very large when the size of $\mathrm{R}$ becomes large, more than $30 \times 30$. There are two kinds of errors; one comes from the truncation error involved in the inversion calculation of the matrix, and another comes from the uncertainties involved in the resolution function. The former can be overcome, but the latter is intrinsic. Therefore, this method is not very useful, as has been discovered by others. 34

Second is the method of incorporating the resolution function in the calculations of the functions used for the common method of least squares. ${ }^{\text {L34 }}$ We have found that this method is satisfactory. The problem of the propagation of truncation errors does not exist here because, statistically, the errors will be averaged. However, the intrinsic error from the resolution function still plays an important role.

We have also tried the method of Fourier transform. We have

$$
y(t)=\int_{-\infty}^{\infty} R\left(t, t^{\prime}\right) Y\left(t^{\prime}\right) d t^{\prime}
$$

From the convolution theorem, we obtain

$$
F(y(t))=F\left(R\left(t, t^{\prime}\right)\right) F\left(Y\left(t^{\prime}\right)\right),
$$


where $\mathrm{F}$ denotes the Fourier transform. We can solve the equation

$$
F\left(Y\left(t^{\prime}\right)\right)=F\left(R\left(t, t^{\prime}\right)\right) / F(y(t))
$$

Then we can obtain $Y\left(t^{\prime}\right)$ using the backward Fourier transform. We have found that this method is similar to the method of matrix inversion the errors of the de-convoluted function $Y\left(t^{\prime}\right)$ are very large.

\section{Instrumentation}

During the early stage of this contract our emphasis was on improving the time resolution of the apparatus measuring the positron annihilation lifetime spectra. We have achieved, a resolution of 200 psec for specific conditions and $<300$ psec for everyday use. ${ }^{L 9, L 12, L 13}$ Subsequent1y, we have not been able to improve the resolution much further. Improvement has been limited by the present state of the art in the manufacturing of scintillators with fast rise time and high light output, and photomultipliers with better focusing systems and fast rise time. Since the number of apparatus used in the positron annihilation studies has not been great enough to support the research and development of this equipment, we can only obtain what is commercially available. Most of this equipment has been produced for the market requirements of research in high energy physics. We hope new developments of faster scintillators and photomultipliers will be able to bring the everyday use resolution down to less than 200 psec. On the other hand, we have also tried other circuits and parts produced by advanced technology. ${ }^{2} 27$ For example, we have made a set of fast coincidence units with a resolution of $8 \mathrm{nsec}$ and a time-to-amplitude converter using Motorola MECL-III integrated circuits. ${ }^{249}$ We have used the coincidence unit in our apparatus for measuring angular correlation and have found it very stable and reliable. 


\section{Data Compilation}

This work started about two years ago when Dr. S. J. Tao, principal investigator, accepted an invitation to be the co-operative U. S. scientist for NSF Grant GF 36834 to Professor R. M. Singru, Indian Institute of Technology, Kanpur, for positron annihilation studies. The work of the grant includes data compilation for positron studies. The last data compilation for positron studies was carried out by the Russian group, headed by Professor Goldanskii, and the Canadian group, headed by Professor Hogg, and published in 1968. It was limited mostly to the data obtained by the method of lifet1me measurement. 59

More than five years have passed since then. There is a need for a recompilation of the data, including all the data published in the field of positron annihilation. It was decided that the data compilation work would involve three parts: (i) a complete list of publications, classified according to the content of the publication; (ii) a list of publications, each with a brief summary, for the data obtained by the method of angular correlation; and (iii) a table for the data obtained by the method of positron annihilation. This work is currently in progress.

\section{Future Outlook and Recommendations}

After being deeply involved in positron annihilation studies and positronium chemistry for the last twelve years, I have the feeling, just like the old saying, the more I have studied the less I know. Put another way, the more I have studied, the more new areas about which very little is known have opened in front of me.

In a general sense, positron annihilation is a simple phenomenon involving a positron and an electron. However, the electron is always 
bound to an atom or a molecule and is not free. Therefore, from the results of annihilation we can assess or evaluate the properties of the electron. The properties may include density, momentum, spin, interactions, etc. The properties directly involved in the process of positron annihilation are more than those involved in photospectroscopy, mass spectroscopy, NMR, ESR, etc.

Positron annihilation has its advantages and disadvantages. The major advantage is that positron annihilation can provide more information. The major disadvantage is that because the content of the information gathered by positron annihilation is greater, it tends to be more diffused and harder to sort out into specifics. This has been the reason for the difficulties which arose on many occasions. The only remedy is more work. However, in the past, the total support given to positron annihilation has been far less than that given to laser, NMR, ESR or others. Even with such financial support, the achievements we (all of the research workers in positron annihilation studies) have made have been substantial, as indicated in the preceeding chapters.

When radioactive isotopes are used directly as the source of positrons for positron studies, positrons act as the primary energetic ionization particles in the medium. The annihilation of "free" positrons, the formation of positronium and the subsequent annihilation of positronium are all a part of the ionization reactions and the hot radical reactions in the realm of radiation chemistry. Actually, in addition to the solvated electron, the positron is one of the few species which can be traced and detected readily during these reactions. We have spent most of the last eight years studying the fundamental properties of the positron and positronium-in order to develop a good foundation for the study of many positron 
and positronium reactions. Probably, it is now time to start some experiments to study various positron and positronium reactions. For example, temperature variation of the positronium reactions with $\mathrm{Cl}_{2}, \mathrm{Br}_{2}$ or $\mathrm{NO}_{2}$, more temperature variations for various quenching agents in inert solvents, angular correlation curves for many systems in both liquid and solid states for the determination of positronium formation in molecular solids and colloidal systems of micells are interesting systems for further studies. As for spectfics, I think more positron studies in phase transition and other transitions of various substances, including polymers or macromolecules, using the methods of both lifetime measurement and angular correlation or Doppler broadening will greatly help our present understanding of the theory of molecular liquids and solids. Positron annihilation is just too sensitive to be discarded as one of the methods for such studies. As a method for the study of defects and other structural changes. in metals and other solids, positrons will occupy a specific position. It is sensitive to many metallic systems, but inert to some of them. The nondestructive nature is an advantage. But some money should be spent for the development of instrumentation, such as faster and more efficient plastic or other scintillators and faster photomultipliers, etc. This will enhance the present work in this area substantially.

The studies using mono-energetic slow positrons will have a special. place in positron annihilation research. In addition to its importance as a pure fundamental branch of science, the surface re-emission of positrons or positronium will be of interest in surface physics or chemistry.

The actual process of the formation of Ps in molecular 1iquids and solids must be solved. Once it is solved, positron annihilation studies 
will contribute tremendously to our present knowledge of radiation chemistry.

Part of my comments on instrumentation concerning the development of better scintillators and photomultipliers has been mentioned before. Certainly, the development of a solid state device with a better energy resolution or a faster time response will also help research in positron annihilation studies greatly.

I also hope the present joint work in data compilation can be finished in 1975 and published in 1976.

I shall not elaborate too much about positron annihilation because there has been so much written during the past five years, as indicated by the number of review articles in many languages and in many countries. I do not think I could add more.

On a world-wide scale, the Russians have contributed tremendously during the last ten years, particularly in two areas--positronium chemistry and defects in metals. Many research workers in Western European countries (with the exception of Spain and Portugal) are doing various kinds of interesting work. Particularly, I would like to mention the work on scattering cross sections of various gases using mono-energetic slow positron beams being performed by the group in University College, London. Many workers in Eastern European countries are starting to set up experiments on positron annihilation. In Asia, most of the work is centered in Japan and India, although there is a possibility some work may be started in Australia again. Possibly China would like to start some work, too.

In North America, Canada still has most of the workers in positron annihilation. And for the U. S., there seems to be a lack of co-ordination among the research workers in positron annihilation and positronium 
chemistry. Probably, it would be worthwhile for either the government or the private sector to sponsor a conference in the U. S. in the near future to bring everyone together. It has been nearly ten years since the last Conference on Positron Annihilation was held in Detroit. Finally, I should express my sincere appreciation to the USAEC and the scientific staff in USAEC for their enthusiasm in supporting this research project in fundamental science. Although I could not attain all the goals I had intended, I have finished much that I wished to accomplish. I would like also to thank many persons who have been involved in this project. Their hard work was essential to the achievements made. 
VI. References

1. F.A.M. Dirac, Proc. Camb. Phil. Soc., 26, 361 (1930).

2. C.D. Anderson, Phys. Rev., 41, 405 (1932); Phys. Rev., 43, 491 (1933); Phys. Rev., 44, 406 (1933).

3. M. Deutsch, M. Prog. Nuc1. Phys., $\underline{3}, 131$ (1953).

4. S. De Benedetti and H. C. Corden, A. Rev. Nucl. Sci., 4, 191 (1954).

5. S. Berko and F.L. Hereford, Rev. Mod. Phys., 28, 299 (1956).

6. R.A. Ferre11, Rev. Mod. Phys., 28, 308 (1956).

7. P.R. Wallace, Adv. Solid St. Phys., 10, 1 (1960).

8. J. H. Green and J. Lee, "Positronium Chemistry," Academic Press, New York - London (1964).

9. "Positron Annihilation," Ed. A.T. Stewart and L. Roellig, Academic Press, New York-London (1967).

10. V.I. Goldanskii, Atomic En. Rev., 6, 3 (1968).

11. Y. Ito and Y. Tabuta, Radioisotopes, 21, 312 (1972).

12. J.A. Merrigan, J.H. Green and S.J. Tao, "Physical Methods of Chemistry, Vo1. I, Part IIID," Ed. A. Weissberger and B.W. Rossiter, John Wiley (1972).

13. H.J. Ache, Angew. Chem. 84, 234 (1972).

14. Bela Levay, Kem. Kozlem. 37, 221 (1972).

15. I. Ya. Dekhtyar, Czech. J. Phys., B18, 1509 (1968).

16. Alfred Seeger, J. Phys. F., $\underline{3}, 248$ (1973).

17. A.E. Hamielec, M. Eldrup. O. Mogensen and P. Jansen, J. Macromol. Sci.-Revs. Macromol. Chem, C9(2), 305-337 (1973).

18. S.J. Tao, J. Be11 and J.H. Green, Nucl. Instr. Meth., 35, 222 (1965).

19. J. Bell, S.J. Tao and J.H. Green, Nucl. Instr. Meth., 35, 213 (1965).

20. A.T. Stewart, Can. J. Phys., 35, 168 (1957).

21. S. Berko and J.S. Plaskett, Pjys. Rev., 112, 1877 (1958). 
22. K. Fujiwara, D. Sueoka and T. Imura, J. Phys. Soc. Japan, 24, 467 (1968).

23. I.K. MacKenzie, J.A. Eady and R.R. Gingerich, Phys. Lett. 33A, 279 (1970).

24. J. Bell, J.C. Celitans, J.H. Green and S.J. Tao, Nucl. Instr. Meth., 37, 51 (1965).

25. K.F. Canter, P.G. Coleman, T.C. Griffith and G.R. Heyland, J. Phys., B5, L167 (1972).

26. P.G. Coleman, T.C. Griffith, G.R. Heyland, Proc. Royal Soc., (London), A331, 561 (1973).

27. S. Marder, V.W. Hughes, C.S. Wu and W. Bennett, Phys. Rev., 103, 1258 (1956).

28. R.E. Bell and R.L. Graham, Phys. Rev., 87, 236 (1952); 90, 644 (1953).

29. F.H.H. Hsu and C.S. Wu, Phys. Rev. Lett., 18, 889 (1967).

30. S.J. Tao, J. Bell and J.H. Green, Proc. Phys. Soc. (London), 83, 453 (1964).

31. D.A.L. Paul, Proc. Phys. Soc. (London), 84, 563 (1964).

32. K.F. Canter, P.G. Coleman, T.C. Griffith and G.R. Heyland, Appl. Phys., 3, 249 (1974).

33. P.G. Coleman, T.C. Griffith and G.R. Heyland, Appl. Phys., 4 , 89 (1974).

34. P.G. Coleman, T.C. Griffith and G.R. Heyland, Private Communication.

35. J.W. Humberston, J. Phys..B., 7, L286 (1974).

36. K.F. Canter and L. Roellig, Phys. Rev. Lett., 25; 328 (1970).

37. J.H. Green and S.J. Tao, J. Chem. Phys., 39, 3160 (1953).

38. J.D. McNutt, W.W. Kinnison and A.D. Ray, J. Chem. Phys., 60, 4730 (1974).

39. S.Y. Chuang and S.J. Tao, Phys. Rev. A, 9, 989 (1974).

40. S.Y. Chuang and S.J. Tao, Appl. Phys., 3, 199 (1974).

41. S.J. Tao, T.M. Kelly and J.M. Wilkenfeld, App1. Phys., 3, 31 (1974).

42. H.J. Ache, Private Communication.

43. A. Ore, Univ. Bergen Arb. Naturvit. rekke., No. 9 (1949). 
44. O.E. Morgensen, J. Chem. Phys., 60, 998 (1974).

45. P.R. Gray, C.F. Cook and G.P. Starm, J. Chem. Phys., 48, 1145 (1968).

46. S.J. Tao, J. Chem.Phys., 56, 5499 (1972).

47. V.I. Goldanskii, V.P. Shantarovich, Ix. Akad. Nauk SSSR. Ser Khim., No. 4, 809 (1970).

48. S.J. Tao and J.H. Green, Proc. Phys. Soc. (London), 85, 463 (1965).

49. J.H. Green and S.J. Tao, Brit. J. App1. Phys., 16, 981 (1965).

50. P. Hautojarvi and E. Pajame, J. Phys. C: Solid State Phys., $\underline{7}$, 3817 (1974).

51. R.M. Singru, Private Communication.

52. S.Y. Chuang and S.J. Tao, J. Phys. Chem., 78, 1261 (1974).

53. J.R. Stevens and A.C. Mao, J. App1. Phys., 41, 4273 (1970).

54. S.Y. Chuang, S.J. Tao and J.M. Wilkenfeld, J. Appl. Phys., 43, 737 (1972).

55. S.Y. Chuang and S.J. Tao, Phys. Lett. 33A, 56 (1970).

56. S.Y. Chuang and S.J. Tao, "Phase Transitions - 1973," Ed. L.E. Cross, Pergamon Press, New York, p. 363 (1973).

57. W.W. Walker and D.C. K1ine, J. Chem. Phys., 60, 4990 (1974).

58. W.W. Walker and E.L. Mueller III, App1. Phys., 3, 155 (1974).

59. B.G. Hogg, G.M. Laidlow, V.I. Goldanskii and V.P. Shantarovich, Atom. Energ. Rev., 6, 149 (1968). 
VII. List of Publications

We are attaching a copy with this report of any publication which appeared in a regular journal. An asterisk marks each of these publications. For some recent publications marked with an extra asterisk, ten copies are attached.

1. NY0-3661-1 "Positronium Chemistry," S. J. Tao, yearly progress report, 1966-67.

2. NY0-3661-2 "Positronium Chemistry," S. J. Tao, yearly progress report, 1967-68.

*3. NYO-3661-3 "Systematic Positronium Chemistry," S. J. Tao and J. H. Green, J. Chem. Soc. (London), A, 408 (1968).

4. NYO-3661-4 "Electron Affinity of Positronium Formation in Solids," S. J. Tao and John Lee, presented to ACS and APS meeting in Hartford, Connecticut, March 17-18, 1967.

5. NY0-3661-5 "The Effect of Minor Impurities in Crystalline Anthracene and Benzene on Positron Annihilation," S. J. Tao and John Lee, Bull. Am. Phys. Soc. 12, 534 (1967).

6. NY0-3661-6 "Positronium Interaction with Iodine in Organic Solvents," S. J. Tao, presented to "Physics and Chemistry of Excited Materials Symposium," University of North Carolina, Chapel Hill, North Carolina, October 1967.

*7. NYO-3661-7 "Methods of Data Reduction in Analyzing Positron Annihilation Lifetime Spectra," S. J. Tao, IEEE, NS-15, 1, 175 (1968).

*8. NY0-3661-8. "Positron Interactions in Aqueous Oxyacids and Hydrogen Compounds," S. J. Tao and J. H. Green, J. Phys. Chem., 73, 882 (1969).

*9. NY0-3661-9 "Recent Developments in Measuring Short Time Intervals by Time-to-Amplitude Converters," A. Ogata, S. J. Tao and J. H. Green, Nuc1. Instr. and Meth., 60, 141 (1968).

10. NY0-3661-10 "Free Radical Scavenger Quenching of Positronium in Liquids," T. M. Kelly and S. J. Tao, Bull. Am. Phys. Soc., 13, 103 (1968).

11. NY0-3661-11 "Computation of Time Dependent Positron Annihilation Rate," S. J. Tao, progress report, 1967. 
*12. NY0-3661-12 "A Time-to-Amplitude Converter of Simple Construction," A. Ogata and S. J. Tao, Nucl. Instr. and Meth., 69, 344 (1969).

13. NYO-3661-13 "A Study on Crossover and Leading Edge Timing Methods," S. J. Tao and A. Ogata, progress report, 1968.

*14. NY0-3661-14 "Theoretical and Experimental Positron Annihilation and Scattering Cross Sections in Helium," S. J. Tao and T. M. Kelly, Phys. Rev. 185, 135 (1969).

15. NY0-3661-15 "o-Positronium Annihilation in Micro-Cavities inside Polypropylene," A. Ogata and S. J. Tao, progress report, 1968. Part of its content appears in NYO-3661-31.

16. NY0-3661-16 "Annihilation of Positronium in a Cavity," S. J. Tao, progress report, 1968. Parts of its content appear in c00-3373-1 and COO-3373-2.

17. NY0-3661-17 "Schroding Equation Solution Program," Harry Miller and S. J. Tao, progress report, 1968.

18. NY0-3661-18 "Iodine Quenching of Positronium in Organic Liquids," T. M. Kelly and S. J. Tao, progress report, 1968. Part of its content appears in NYO-3661-22.

19. NY0-3661-19. "Positron Annihilation in Helium Gas at $295^{\circ} \mathrm{K}, "$ T. M. Kelly and S. J. Tao, progress report, 1969.

20. NYO-3661-20 "Positronium Chemistry," S. J. Tao, yearly progress report, 1968-69.

*21. NY0-3661-21 "Study of Inhibition and Quenching of Positronium by Iodine and $\mathrm{CCl}_{4}$," S. Y. Chuang and S. J. Tao, J. Chem. Phys., 52, 749 (1970).

*22. NY0-3661-22 "Positronium and Iodine Reactions in Organic Solvents," S. J. Tao, J. Chem. Phys., 52, 752 (1970).

*23. NY0-3661-23 "Annihilation of Positrons in Argon at High Densities," S. J. Tao, Phys. Rev. A, 1, 1257 (1970).

24. NYO-3661-24 "Positronium Annihilation in Silica Gels," S. Y. Chuang and S. J. Tao, Bull. Am. Phys. Soc. 14, 89 (1969).

25. NYO-3661-25. "Positron Lifetimes in Polymers of Various Molecular Weights," S. J. Tao and S. Y. Chuang, Bull. Am. Phys. Soc., 14, 407 (1969).

*26. NY0-3661-26 "Annihilation Positrons in Nitrogen," S. J. Tao, Phys. Rev. A, 2, 1669 (1970); Bull. Am. Phys. Soc. 14 (1969). 
27. NY0-3661-27 "A Fast-Fast Coincidence Dual Range Time-to-Amplitude Converter," S. J. Tao, progress report, 1969. Part of the work is included in NYO-3661-26.

28. NY0-3661-28 "Annihilation of Ortho-Positronium in Large Cavities," S. Y. Chuang and S. J. Tao, Bull. Am. Phys. Soc. 15, 57 (1970).

29. NYo-3661-29 "Positron Chemistry," S. J. Tao, progress report, not for public announcement or distribution.

*30. NY0-3661-30 "Positron Lifetimes in Shock-Deformed Polycrystalline Nickel," W. H. Holt, M. F. Rose, S. Y. Chuang and S. J. Tao, Phys. Lett. A, 32A, 422 (1970).

*31. NY0-3661-31 "Ortho-Positronium Annihilation in Nitric Acid Treated Polypropylene," A.' Ogata and S. J. Tao, J. Appl. Phys., 41, 4261 (1970).

*32. NYO-3661-32 "Temperature and Phase Dependence of Positron Lifetimes in $\mathrm{CH}_{3} \mathrm{OG}$ and $\mathrm{CD}_{3} \mathrm{OD}, " \mathrm{~S}$. Y. Chuang and $\mathrm{S}$. J. Tao, Phys. Lett. A, 33A, 56 (1970).

33. NYo-3661-33 "A Preliminary Study on the Temperature Effect on Positron Annihilation in $\mathrm{KClO}_{4}, " \mathrm{~S}$. J. Tao and S. Y. Chuang, progress report, 1970.

34. NY0-3661-34 "Non-Linear Least Squares Fit to Multi-exponential Component Curves - Date Reduction Programs for Positron Annihilation Lifetime Spectra," Marcia Hyde and S. J. Tao, progress report, 1970.

35. NY0-3661-35 "The Effect of Temperature on the Mean Life of o-Positronium in Silica Gels," S. Y. Chuang and S. J. Tao, Bull. Am. Phys. Soc., 15, 812 (1970). Part of the content appears in C00-3373-9.

36. NY0-3661-36 "Temperature and Phase Dependence of Positron Lifetimes in Methyl Mercaptan," S. Y. Chuang and S. J. Tao, Bull. Am. Phys. Soc., 16, 64 (1971). A part of it appears in C00-3373-10.

*37. NY0-3661-37 "Study of Various Properties of Silica Gel by Positron Annihilation," S. Y. Chuang and' S. J. Tao, J. Chem. Phys., 34, 4902, (1971).

38. NYO-3661-38 "Positronium Lifetime Measurements in NO and $\mathrm{NO}_{2}$," J. M. Wilkenfeld, S. J. Tao and S. Y. Chuang, Bull. Am. Phys. Soc., 16, 618 (1971). The content of this paper has been greatly revised and published as coo-3373-2.

*39. NY0-3661-39 "Ortho-Positronium Annihilation and the Glass Transition of Nylon 06," S. Y. Chuang, S. J. Tao and J. M. Wilkenfeld, J. Appl. Phys., 43, 737 (1972). 
40. NYO-3661-40 "The Adsorption of o-Ps on Solid Surfaces," S. Y. Chuang, S. J. Tao and J. M. Wilkenfeld, International Positron Annihilation Conference, Kingston, Ontario, August 1971.

41. NY0-3661-41 "Positron Formation in Helium," Margaret F. Fels and S. J. Tao. Due to the moving of Margaret F. Fels, correspondence with her has been difficult. We are still hoping the paper can be sent out for publication.

42. NYO-3661-42 "Positronium Chemistry," S. J. Tao. This is the progress report of the year 1971 .

*43. Co0-3373-1 "Positronium Annihilation in Molecular Substances," S. J. Tao, J. Chem. Phys., 56, 5499 (1972).

*44. COO-3373-2 "POSItRON ANNIHILATION", J. A. Merrigan, J. H. Green and S. J. Tao, "Physical Methods of Chemistry," Vol. I, Part IIID, Ed., Weissberger and Rossiter, John Wiley, (1972). Copy attached.

*45. Co0-3373-3 "Positron Annihilation in Nitric Oxide and Nitrogen. Dioxide," S. J. Tao, S. Y. Chuang and J. Wilkenfeld, Phys. Rev. A 6, 1967 (1972).

**46. C00-3373-4 "Positronium Diffusion and 1ts Reactions in Solutions," S. J. Tao, T. M. Kelly, S. Y. Chuang and J. M. Wilkenfeld, presented to the Third International Conference of Positron Annihilation, Helsinki, August 1973, Appl. Phys., 3 , 31 (1974).

**47. Co0-3373-5 "Positronium Annihilation and Average Molecular Weight of Polymers," S. Y. Chuang and S. J. Tao, J. App1. Phys., 44, 5171 (1973).

**48. C00-3373-6 "Positronium Annihilation in Amino Acids and Proteins," S. Y. Chuang and S. J. Tao, presented to A.P.S., Bull. Am. Phys. Soc., 4, 588 (1973); J. Phys. Chem., 78, 1261 (1974).

*49. Co0-3373-7. "A Simple Time-to-Amplitude Converter and a Quadruple Coincidence Unit Based on Integrated-Circuits for Positron Annihilation Studies," A. Ogata, J. B. Wang, S. Y. Chuang, and S. J. Tao, Nucl. Instr. and Meth., 108, 253 (1973).

50. C00-3373-8 "Positronium Chemistry," S. J. Tao, progress report, 1972.

*51. C00-3373-9 "Correlation of o-Ps Annihilation and the Surface Properties of Silica Gels as a Function of Temperature," S. Y. Chuang and S. J. Tao, Can. J. Phys., 51, 820 (1973).

**52. Co0-3373-10 "Detection of Phase Transitions in Molecular Crystals and Macromolecular Substances by Positron Annihilation," S. Y. Chuang and S. J. Tao, presented to the Conference on Phase Transitions and their Applications in Materials Sciences, May 1973, Penn. State Univ., "Phase Transitions - 1973," Ed. L. E. Cross, Pergamon Press, New York, 1974. 
**53. C00-3373-11 "Bromine and Oxygen Quenching of Positronium in Silica Gel," S. Y. Chuang and S. J. Tao, presented to the Third International Conference of Positron Annihilation, Helsinki, August 1973; App1. Phys., 3 , 199-204 (1974).

**54. Co0-3373-12 "Positronium in Molecular Substances," S. J. Tao, An invited review paper, presented to the Third International Conference of Positron Annihilation, Helsinki, August 1973; App1. Phys. $\underline{3}, 1$ (1974).

**55. Co0-3373-13 "Quenching of Positronium in Nitrogen Dioxide," S. Y. Chuang and S. J. Tao, Phys. Rev. A, 9 , 989 (1974).

56. Co0-3373-14 "Positronium Annihilation and Critical Surface Tension," S. J. Tao and S. Y. Chuang, progress report, 1974.

57. C00-3373-15 "Positron-Dislocation Interaction: for Non-destructive Evaluation," J. P. Wallace, J. K. Tien, S. J. Tao and S. Y. Chuang, submitted to Materials Science Congress, Chicago, October 1973.

58. co0-3373-16 "Positronium Chemistry," S. J. Tao, progress report of this year.

**59. Co0-3373-17 "A Long Lifetime Correlation with Plastic Deformation in Metals and Alloys," J. P. Wallace, J. K. Tien, S. J. Tao and S. Y. Chuang, Scripta Metallurgica, 8,1039 (1974). 